\title{
Article
}

\section{The Spectrum of COVID-19-Associated Myocarditis: A Patient-Tailored Multidisciplinary Approach}

\author{
Giovanni Peretto ${ }^{1,2,3, *}$, Andrea Villatore ${ }^{3}$ (), Stefania Rizzo ${ }^{4}$, Antonio Esposito ${ }^{2,3,5}$, Giacomo De Luca ${ }^{2,6}$, \\ Anna Palmisano ${ }^{2,5}$, Davide Vignale ${ }^{2,5}$, Alberto Maria Cappelletti ${ }^{7}$, Moreno Tresoldi ${ }^{8,9}$,

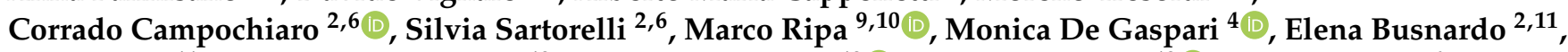 \\ Paola Ferro ${ }^{11}$, Maria Grazia Calabrò ${ }^{12}$, Evgeny Fominskiy ${ }^{12}{ }^{(0}$, Fabrizio Monaco ${ }^{12}{ }^{\circledR}$, Giulio Cavalli ${ }^{6}$, \\ Luigi Gianolli ${ }^{11}$, Francesco De Cobelli ${ }^{3,5}{ }^{10}$, Alberto Margonato ${ }^{7}$, Lorenzo Dagna ${ }^{3,6}$, Mara Scandroglio ${ }^{12}$, \\ Paolo Guido Camici ${ }^{3}$, Patrizio Mazzone ${ }^{1}$, Paolo Della Bella ${ }^{1}$, Cristina Basso ${ }^{4}$ (1) and Simone Sala ${ }^{1,2}$
}

Citation: Peretto, G.; Villatore, A.; Rizzo, S.; Esposito, A.; De Luca, G.; Palmisano, A.; Vignale, D.;

Cappelletti, A.M.; Tresoldi, M.;

Campochiaro, C.; et al. The Spectrum of COVID-19-Associated Myocarditis A Patient-Tailored Multidisciplinary Approach. J. Clin. Med. 2021, 10, 1974. https://doi.org/10.3390/ jcm10091974

Academic Editor: Andrea Igoren Guaricci

Received: 17 March 2021

Accepted: 22 April 2021

Published: 4 May 2021

Publisher's Note: MDPI stays neutral with regard to jurisdictional claims in published maps and institutional affiliations.

Copyright: (c) 2021 by the authors. Licensee MDPI, Basel, Switzerland. This article is an open access article distributed under the terms and conditions of the Creative Commons Attribution (CC BY) license (https:// creativecommons.org/licenses/by/ $4.0 /)$
1 Department of Cardiac Electrophysiology and Arrhythmology, IRCCS San Raffaele Scientific Institute, 20132 Milan, Italy; mazzone.patrizio@hsr.it (P.M.); dellabella.paolo@hsr.it (P.D.B.); sala.simone@hsr.it (S.S.)

2 Myocarditis Disease Unit, IRCCS San Raffaele Scientific Institute, 20132 Milan, Italy; esposito.antonio@unisr.it (A.E.); deluca.giacomo@hsr.it (G.D.L.); palmisano.anna@hsr.it (A.P.); vignale.davide@hsr.it (D.V.); campochiaro.corrado@hsr.it (C.C.); sartorelli.silvia@hsr.it (S.S.); busnardo.elena@hsr.it (E.B.)

3 School of Medicine, San Raffaele Vita-Salute University, 20132 Milan, Italy; a.villatore@studenti.unisr.it (A.V.); decobelli.francesco@hsr.it (F.D.C.); dagna.lorenzo@unisr.it (L.D.); camici.paolo@hsr.it (P.G.C.)

4 Department of Cardiac Thoracic Vascular Sciences and Public Health, Cardiovascular Pathology, Padua University, 35128 Padua, Italy; s.rizzo@unipd.it (S.R.); monica.deg1@gmail.com (M.D.G.); cristina.basso@unipd.it (C.B.)

5 Experimental Imaging Center, Radiology Unit, IRCCS San Raffaele Scientific Institute, 20132 Milan, Italy

6 Unit of Immunology, Rheumatology, Allergy and Rare Diseases (UnIRAR), IRCCS San Raffaele Scientific Institute, 20132 Milan, Italy; cavalli.giulio@hsr.it

7 Department of Clinical Cardiology and Intensive Care Unit, IRCCS San Raffaele Scientific Institute, 20132 Milan, Italy; cappelletti.alberto@hsr.it (A.M.C.); margonato.alberto@hsr.it (A.M.)

8 Unit of General Medicine and Advanced Care, IRCCS San Raffaele Scientific Institute, 20132 Milan, Italy; tresoldi.moreno@hsr.it

9 COVID-19 Unit, IRCCS San Raffaele Scientific Institute, 20132 Milan, Italy; ripa.marco@hsr.it

10 Department of Infectious Diseases, IRCCS San Raffaele Scientific Institute, 20132 Milan, Italy

11 Department of Nuclear Medicine, IRCCS San Raffaele Scientific Institute, 20132 Milan, Italy; ferro.paola@hsr.it (P.F.); gianolli.luigi@hsr.it (L.G.)

12 Cardiac Surgery Intensive Care Unit, Division of Anesthesiology, IRCCS San Raffaele Scientific Institute, 20132 Milan, Italy; calabro.mariagrazia@hsr.it (M.G.C.); fominskiy.evgeny@hsr.it (E.F.); monaco.fabrizio@hsr.it (F.M.); scandroglio.mara@hsr.it (M.S.)

* Correspondence: peretto.giovanni@hsr.it; Tel./Fax: +39-02-2643-7484-7326

\begin{abstract}
Background. Myocarditis lacks systematic characterization in COVID-19 patients. Methods. We enrolled consecutive patients with newly diagnosed myocarditis in the context of COVID-19 infection. Diagnostic and treatment strategies were driven by a dedicated multidisciplinary disease unit for myocarditis. Multimodal outcomes were assessed during prospective follow-up. Results. Seven consecutive patients ( $57 \%$ males, age $51 \pm 9$ y) with acute COVID-19 infection received a de novo diagnosis of myocarditis. Endomyocardial biopsy was of choice in hemodynamically unstable patients ( $n=4$, mean left ventricular ejection fraction (LVEF) $25 \pm 9 \%$ ), whereas cardiac magnetic resonance constituted the first exam in stable patients $(n=3$, mean LVEF $48 \pm 10 \%$ ). Polymerase chain reaction (PCR) analysis revealed an intra-myocardial SARS-CoV-2 genome in one of the six cases undergoing biopsy: in the remaining patients, myocarditis was either due to other viruses $(n=2)$ or virus-negative $(n=3)$. Hemodynamic support was needed for four unstable patients $(57 \%)$, whereas a cardiac device implant was chosen in two of four cases showing ventricular arrhythmias. Medical treatment included immunosuppression (43\%) and biological therapy (29\%). By the 6-month median follow-up, no patient died or experienced malignant arrhythmias. However, two cases (29\%) were screened for heart transplantation. Conclusions. Myocarditis associated with acute COVID-19 infection is a spectrum of clinical manifestations and underlying etiologies. A multidisciplinary approach is the cornerstone for tailored management.
\end{abstract}


Keywords: myocarditis; COVID-19; SARS-CoV-2; ventricular arrhythmias; endomyocardial biopsy; cardiac magnetic resonance; multidisciplinary; immunosuppression; inflammation

\section{Introduction}

Among a wide range of cardiovascular manifestations, myocarditis is a possible complication of the severe acute respiratory syndrome coronavirus-2 (SARS-CoV-2) infection responsible for the current COVID-19 pandemic [1,2]. Due to the lack of investigation by endomyocardial biopsy (EMB) and cardiac magnetic resonance (CMR), however, in many published reports, myocarditis was just clinically suspected and largely undistinguishable from other causes of myocardial injury [1,3]. In turn, treatment strategies and outcomes of COVID-19-associated myocarditis are still to be defined. We report the first series of patients diagnosed with acute SARS-CoV-2 infection undergoing advanced diagnostic characterization and multidisciplinary management for associated myocarditis, proven by gold standard diagnostic techniques. In particular, we aimed at describing the wide spectrum of COVID-19-associated myocarditis, and the results of a patient-tailored management by a dedicated "myocarditis disease unit" [4].

\section{Materials and Methods}

Consecutive patients with clinically suspected myocarditis in the context of acute SARS-CoV-2 infection were enrolled from March 2020 to December 2020 at a third-level referral center for COVID-19 management. As a part of an internal protocol, approved by the local institutional review board, written informed consent was obtained from all patients. Diagnostic and therapeutic workup and uniform study endpoints are reported in Online Supplements.

\section{Results}

Seven consecutive patients $(0.2 \%$ of the COVID-19 inpatients during the enrollment period) were diagnosed with myocarditis. Clinical features of every single case are presented in the following section. Baseline characteristics of the whole series are summarized in Table 1, whereas treatment strategies and study endpoints are reported in Table 2.

Table 1. Baseline features and diagnostic workup.

\begin{tabular}{|c|c|c|c|c|c|c|c|}
\hline Features & P1 & P2 & P3 & $\mathrm{P} 4$ & P5 & P6 & P7 \\
\hline \multicolumn{8}{|c|}{ General features } \\
\hline Age, $y$ & 43 & 38 & 58 & 45 & 64 & 50 & 56 \\
\hline Gender & female & male & female & male & male & male & female \\
\hline Ethnicity & $\begin{array}{c}\text { African } \\
\text { American }\end{array}$ & $\begin{array}{c}\text { African } \\
\text { American }\end{array}$ & Caucasian & Caucasian & Caucasian & Caucasian & Caucasian \\
\hline COVID diagnosis & NPS & NPS & NPS & $\begin{array}{c}\text { NPS, BAS, } \\
\text { BAL }\end{array}$ & NPS & NPS & NPS \\
\hline Pneumonia diagnosis & $\mathrm{XR}, \mathrm{CT}$ & $\mathrm{XR}, \mathrm{CT}$ & $\mathrm{XR}, \mathrm{CT}$ & $\mathrm{XR}, \mathrm{CT}$ & negative & $\mathrm{XR}, \mathrm{CT}$ & negative \\
\hline $\begin{array}{c}\text { Delay from first cardiac } \\
\text { abnormality to } \\
\text { pneumonia, days }\end{array}$ & 0 & 6 & 0 & 8 & no & 12 & no \\
\hline \multicolumn{8}{|c|}{ Clinical presentation } \\
\hline Myocarditis presentation & ACS-like & $\mathrm{HF}$ & ACS-like & $\mathrm{HF}$ & VA & $\mathrm{HF}$ & $\begin{array}{c}\text { ACS-like, } \\
\text { HF }\end{array}$ \\
\hline Sp02, \% & 89 & 96 & 96 & 88 & 81 & 88 & 76 \\
\hline $\mathrm{T},{ }^{\circ} \mathrm{C}$ & 37.7 & 36.5 & 37.0 & 38.5 & 38.7 & 37.1 & 36.2 \\
\hline
\end{tabular}


Table 1. Cont.

\begin{tabular}{|c|c|c|c|c|c|c|c|}
\hline Features & P1 & $\mathrm{P} 2$ & P3 & $\mathrm{P} 4$ & P5 & P6 & P7 \\
\hline \multicolumn{8}{|c|}{ Clinical presentation } \\
\hline Prodromes & fever, vomit & cough & $\begin{array}{l}\text { fever, vomit, } \\
\text { diarrhea }\end{array}$ & $\begin{array}{l}\text { fever, vomit, } \\
\text { diarrhea }\end{array}$ & no & cough & fever \\
\hline $\begin{array}{c}\text { Delay from prodromes } \\
\text { to cardiac symptoms, } \\
\text { days }\end{array}$ & 2 & 7 & 7 & 5 & 0 & 10 & 12 \\
\hline $\begin{array}{l}\text { Coronary artery } \\
\text { assessment }\end{array}$ & $\mathrm{CT}$ & no & $\mathrm{CT}$ & $\mathrm{CT}$ & CA & CA & CA \\
\hline \multicolumn{8}{|c|}{ Blood exams } \\
\hline WBC peak, $10^{6} / \mathrm{ml}$ & 18.9 & 8.7 & 4.4 & 19.8 & 28.6 & 9.0 & 28.7 \\
\hline CRP peak, mg/L & 21 & 208 & 52 & 309 & 233 & 12 & 79 \\
\hline T-Tn peak, ng/L & 135 & 26 & 222 & 39 & 487 & 29 & 13,722 \\
\hline $\begin{array}{c}\text { NTproBNP peak, } \\
\mathrm{pg} / \mathrm{mL}\end{array}$ & 1001 & 148 & 261 & 24,252 & 1170 & 1122 & 15,131 \\
\hline $\begin{array}{l}\text { Screening for } \\
\text { autoimmunity }\end{array}$ & $\begin{array}{c}\text { AHA, } \\
\text { anti-TPO }\end{array}$ & AHA & normal & ACL IgG & $\begin{array}{r}\text { AHA, } \\
\text { ANCA }\end{array}$ & normal & AHA \\
\hline \multicolumn{8}{|c|}{ Electrocardiogram (ECG) and arrhythmias } \\
\hline Presentation rhythm & atrial ectopic & sinus & sinus & sinus & VF & sinus & sinus \\
\hline $\mathrm{PQ}, \mathrm{ms}$ & 170 & 159 & 147 & 172 & 167 & 216 & 192 \\
\hline QRS, ms & 94 & 90 & 82 & 98 & 82 & 105 & 130 \\
\hline QTc, md & 452 & 444 & 392 & 378 & 421 & 456 & 445 \\
\hline Abnormal ST & yes & no & yes & no & yes & no & yes \\
\hline Abnormal T-waves & yes & yes & yes & no & yes & yes & yes \\
\hline BA & $\begin{array}{c}\text { AJR } \\
\text { (self-limited) }\end{array}$ & no & no & - & - & $\begin{array}{c}\text { 1st-degree } \\
\text { AVB }\end{array}$ & $\begin{array}{l}\text { 3rd-degree } \\
\text { AVB, LBBB }\end{array}$ \\
\hline SVA & no & no & no & - & - & - & $\mathrm{AF}$ \\
\hline VA & no & no & no & PVC, NSVT & $\begin{array}{l}\text { VF, NSVT, } \\
\text { PVC }\end{array}$ & PVC, NSVT & NSVT, VT \\
\hline \multicolumn{8}{|c|}{ Echocardiogram and CMR } \\
\hline LVEDVi, $\mathrm{mL} / \mathrm{m}^{2}$ & 52 & 61 & 47 & 77 & 82 & 115 & 44 \\
\hline LVEF, \% & 43 & 41 & 59 & 34 & 15 & 20 & 30 \\
\hline $\begin{array}{c}\text { Dominant WMA } \\
\text { localization }\end{array}$ & $\begin{array}{l}\text { IL, reverse } \\
\text { TTS }\end{array}$ & AS & no & diffuse & diffuse & IL & AS \\
\hline TAPSE, mm & 18 & 18 & 22 & 12 & 14 & 23 & 8 \\
\hline RVEDD, mm & 27 & 26 & 25 & 38 & 32 & 40 & 50 \\
\hline CMR timing, day & 7 & 8 & 7 & 99 & 13 & - & 17 \\
\hline LGE & no & no & $\begin{array}{l}\text { mid-basal AS } \\
\text { (subepicar- } \\
\text { dial) }\end{array}$ & 0 & $\begin{array}{l}\text { mid-basal } \\
\text { IL (subepi- } \\
\text { cardial) }\end{array}$ & - & $\begin{array}{c}\text { basal septal } \\
\text { (midwall), } \\
\text { RV }\end{array}$ \\
\hline STIR & $\begin{array}{l}\text { mid-basal } \\
\text { diffuse }\end{array}$ & $\begin{array}{l}\text { diffuse } \\
\text { basal, } \\
\text { mid } \\
\text { septal }\end{array}$ & AS & diffuse & $\begin{array}{l}\text { mid-basal } \\
\text { IL }\end{array}$ & - & $\begin{array}{l}\text { mid-basal } \\
\text { septal, RV }\end{array}$ \\
\hline $\mathrm{T} 1 \mathrm{max}, \mathrm{ms}$ & 1234 & 1125 & 1311 & 1055 & 1159 & - & 1232 \\
\hline $\mathrm{T} 2 \mathrm{max}, \mathrm{ms}$ & 67 & 58 & 62 & 54 & 67 & - & 65 \\
\hline Pericardial effusion & No & mild & no & mild & mild & - & mild \\
\hline
\end{tabular}


Table 1. Cont.

\begin{tabular}{|c|c|c|c|c|c|c|c|}
\hline Features & P1 & $\mathrm{P} 2$ & P3 & P4 & P5 & P6 & P7 \\
\hline \multicolumn{8}{|c|}{ EMB } \\
\hline EMB timing, day & 7 & 9 & - & 17 & 9 & 118 & 7 \\
\hline Edema & Yes & yes & - & yes & yes & yes & yes \\
\hline $\begin{array}{l}\text { Inflammatory } \\
\text { infiltrates }\end{array}$ & yes & yes & - & yes & yes & yes & yes \\
\hline $\mathrm{CD} 3+>7 / \mathrm{mm}^{2}$ & yes & no & - & yes & yes & yes & yes \\
\hline $\mathrm{CD} 68+>4 / \mathrm{mm}^{2}$ & no & yes & - & yes & no & no & no \\
\hline Necrosis & mild & no & - & no & no & no & massive \\
\hline $\begin{array}{l}\text { Replacement } \\
\text { fibrosis }\end{array}$ & no & mild & - & mild & no & mild & no \\
\hline Viral genome & no & $\begin{array}{c}\text { PVB19 (low-load), } \\
\text { SARS-CoV2 } \\
(\mathrm{N}+/ \text { ORF1-) }\end{array}$ & - & $\begin{array}{c}\text { PVB19 } \\
\text { (high-load) }\end{array}$ & no & no & $\begin{array}{c}\text { EBV } \\
\text { (high-load) }\end{array}$ \\
\hline
\end{tabular}

Complete features of patients (P1-P7) and baseline diagnostic workup are shown. ACL = anti-cardiolipin; ACS = acute coronary syndrome; $\mathrm{AF}=$ atrial fibrillation; $\mathrm{AHA}=$ anti-heart autoantibodies; $\mathrm{AJR}=$ accelerated junctional rhythm; $\mathrm{ANCA}=$ anti-neutrophil cytoplasmatic autoantibodies; $\mathrm{AS}=$ anteroseptal; $\mathrm{AVB}=$ atrioventricular block; $\mathrm{BA}=$ bradyarrhythmias; $\mathrm{BAL}=$ bronchoalveolar lavage; $\mathrm{BAS}=$ bronchoaspirate; $\mathrm{CA}=$ coronary angiography; $\mathrm{CD}=$ cluster of differentiation; $\mathrm{CMR}=$ cardiac magnetic resonance; $\mathrm{CRP}=\mathrm{C}$-reactive protein (n.v. $<6 \mathrm{mg} / \mathrm{L}$ ); $\mathrm{CT}=$ computed tomography; $\mathrm{EBV}=$ Epstein-Barr virus; $\mathrm{EMB}=$ endomyocardial biopsy; $\mathrm{HF}=$ heart failure; $\mathrm{IL}=$ inferolateral; $\mathrm{LBBB}=$ left bundle branch block; LGE = late gadolinium enhancement; LVEDVi = left ventricular end-diastolic volume (indexed); LVEF = left ventricular ejection fraction; NPS = nasopharyngeal swab; NSVT = nonsustained ventricular tachycardia; NTproBNP $=\mathrm{N}$-terminal pro-brain natriuretic pepetide (n.v. $<88 \mathrm{pg} / \mathrm{mL}$ ); PVB19 = parvovirus B19; $\mathrm{PVC}=$ premature ventricular complexes; RVEDD = right ventricular end-diastolic diameter (RV2); SARS-CoV2 = severe acute respiratory syndrome coronavirus-2 (N and ORF1 genes); STIR = short-tau inversion recovery; SVA = supraventricular arrhythmias; T = temperature; T-Tn $=\mathrm{T}$-troponin $(\mathrm{n} . \mathrm{v} .<14 \mathrm{ng} / \mathrm{L}) ;$ T1/T2 = T-mapping sequences (T1 n.v. < $1045 \mathrm{~ms} ; \mathrm{T} 2$ n.v. $<50 \mathrm{~ms}$ ); TAPSE = tricuspid annular plane systolic excursion; TPO = tyroid peroxydase; $\mathrm{VA}=$ ventricular arrhythmias; $\mathrm{VF}=$ ventricular fibrillation; $\mathrm{VT}=$ ventricular tachycardia; $\mathrm{WBC}=$ white blood cells; $\mathrm{WMA}=$ wall motion abnormalities; $\mathrm{XR}=\mathrm{X}$-ray.

Table 2. Treatment and follow-up.

\begin{tabular}{|c|c|c|c|c|c|c|c|}
\hline Features & P1 & $\mathrm{P} 2$ & P3 & $\mathrm{P} 4$ & P5 & $\mathrm{P} 6$ & P7 \\
\hline \multicolumn{8}{|c|}{ Acute-phase treatment } \\
\hline ICU stay, days & 0 & 0 & 2 & 7 & 5 & 0 & 24 \\
\hline Iv diuretics & yes & yes & no & yes & yes & yes & yes \\
\hline Inotropes & no & no & no & Adr, Nor, Lev & Adr, Nor & dopamine & Adr, Nor, Lev \\
\hline MCS & no & no & no & IABP & no & no & $\begin{array}{c}\text { IABP, } \\
\text { VA-ECMO }\end{array}$ \\
\hline Mechanical ventilation & no & no & no & yes & no & no & yes \\
\hline Cardiac devices & no & no & no & no & ICD & S-ICD & $\begin{array}{l}\text { temporary } \\
\text { PM }\end{array}$ \\
\hline $\begin{array}{l}\text { Etiology-driven } \\
\text { treatment }\end{array}$ & $\begin{array}{l}\mathrm{HCQ}, \\
\mathrm{L} / \mathrm{R}\end{array}$ & $\mathrm{AZT}$ & HCQ & HCQ, AKR & no & $\begin{array}{c}\text { HCQ, AZT, AKR, PDN } \\
+ \text { AZA }\end{array}$ & PDN, IVIG \\
\hline \multicolumn{8}{|c|}{ Discharge assessment } \\
\hline Hospitalization, days & 13 & 35 & 11 & 31 & 19 & 36 & 24 \\
\hline Symptoms & no & no & $\begin{array}{l}\text { mild } \\
\text { chest } \\
\text { pain }\end{array}$ & no & dyspnea & dyspnea & dyspnea \\
\hline NYHA class & I & I & I & II & II & III & II \\
\hline $\mathrm{T}-\mathrm{Tn}, \mathrm{ng} / \mathrm{L}$ & 11 & 10 & 5 & 13 & 24 & 8 & 1416 \\
\hline NTproBNP, pg/ml & 76 & 136 & 261 & 121 & 358 & 383 & 1937 \\
\hline LVEF, \% & 65 & 61 & 60 & 45 & 40 & 20 & 65 \\
\hline TAPSE, mm & 20 & 22 & 24 & 16 & 18 & 20 & 10 \\
\hline
\end{tabular}


Table 2. Cont.

\begin{tabular}{|c|c|c|c|c|c|c|c|}
\hline Features & P1 & P2 & P3 & $\mathrm{P} 4$ & P5 & P6 & P7 \\
\hline \multicolumn{8}{|c|}{ Discharge assessment } \\
\hline RAAS inhibitors & no & no & no & ramipril & enalapril & valsartan & no \\
\hline Betablockers & no & no & bisoprolol & metoprolol & metoprolol & bisoprolol & no \\
\hline Antiarrhythmics & no & no & no & no & no & AMD & ivabradin \\
\hline Diuretics & no & no & SL & FS/SL & FS/SL & FS/SL & FS/CR \\
\hline \multicolumn{8}{|c|}{ Follow-up assessment } \\
\hline $\begin{array}{l}\text { Last follow-up, } \\
\text { months }\end{array}$ & 9 & 6 & 6 & 6 & 6 & 6 & 3 \\
\hline Follow-up mode & $\begin{array}{l}\text { IP, } \\
\text { TM }\end{array}$ & $\mathrm{TM}$ & IP, TM & IP & IP, TM & IP, TM & $\mathrm{TM}$ \\
\hline Death & no & no & no & no & no & no & no \\
\hline Arrhythmias & no & no & no & no & PVC & PVC, NSVT & $\begin{array}{c}\text { PVC, NSVT, } \\
\text { AF }\end{array}$ \\
\hline End-stage heart failure & no & no & no & no & no & referred for HTx & $\begin{array}{c}\text { referred for } \\
\text { HTx }\end{array}$ \\
\hline New hospitalization & no & no & no & no & no & no & no \\
\hline Symptoms & no & no & $\begin{array}{l}\text { dyspnea } \\
\text { on effort }\end{array}$ & no & no & dyspnea & dyspnea \\
\hline NYHA class & I & I & II & I & I & III & II \\
\hline $\mathrm{T}-\mathrm{Tn}, \mathrm{ng} / \mathrm{L}$ & 5 & 6 & 6 & 8 & 13 & 6 & 32 \\
\hline NTproBNP, pg/ml & 56 & 88 & 154 & 76 & 103 & 272 & 887 \\
\hline LVEF, \% & 64 & 63 & 61 & 47 & 60 & 24 & 63 \\
\hline TAPSE, mm & 20 & 21 & 25 & 25 & 20 & 20 & 12 \\
\hline
\end{tabular}

Tailored treatment strategies and outcomes are shown for all patients (P1-P7). AF = atrial fibrillation; Adr = adrenaline; AKR = anakinra; $\mathrm{AMD}=$ amiodarone; $\mathrm{AZA}$ = azathioprine; $\mathrm{AZT}$ = azithromycine; $\mathrm{CR}=$ canrenoate; $\mathrm{FS}$ = furosemide; $\mathrm{HCQ}=$ hydroxychloroquine; $\mathrm{HTx}=$ heart transplantation; IABP = intra-aortic balloon pump; ICD = implantable cardioverter defibrillator; ICU = intensive care unit; $\mathrm{IP}=$ in-person; IVIG = intravenous immunoglobulins; $\mathrm{L} / \mathrm{R}=$ lopinavir/ritonavir; Lev = levosimendan; $\mathrm{LVEF}=$ left ventricular ejection fraction; MCS = mechanical circulatory support; Nor = noradrenaline; NSVT = nonsustained ventricular tachycardia; NTproBNP = N-terminal pro-brain natriuretic pepetide (n.v. $<88$ pg $/ \mathrm{mL}$ ); NYHA = New York Heart Association; PDN = prednisone; $\mathrm{PM}=$ pacemaker; $\mathrm{PVC}$ = premature ventricular complexes; RAAS = renin-angiotensin-aldosterone system; $\mathrm{S}-\mathrm{ICD}=$ subcutaneous ICD; $\mathrm{SL}=$ spironolactone; T-Tn = T-troponin (n.v. $<14 \mathrm{ng} / \mathrm{L}$ ); TM = telemedicine; TAPSE = tricuspid annular plane systolic excursion; VA-ECMO = venoarterial extracorporeal membrane oxygenator.

\subsection{Patient 1 (P1)}

A 43-year-old woman was admitted to hospital for epigastric pain. The nasopharyngeal swab (NPS) was positive for SARS-CoV-2, and the computed tomography (CT) scan showed bilateral pneumonia. The electrocardiogram (ECG) had mild ST-segment elevation and transitory phases of an accelerated junctional rhythm (Figure 1). The left ventricular ejection fraction (LVEF) was 43\%, and both T-troponin (T-Tn) and NTproBNP were elevated. Coronary artery disease was ruled out by CT scan. Cardiac magnetic resonance (CMR) showed diffuse edema and a reverse takotsubo kinetic pattern. Endomyocardial biopsy (EMB) was performed at bedside and showed virus-negative lymphocytic myocarditis with minimal necrosis. Serum anti-heart autoantibodies (AHA) tested positive. Since early recovery was observed (final LVEF 65\%, absent arrhythmias, normal T-Tn), the patient was discharged with no therapy. The nine-month follow-up was uneventful. 


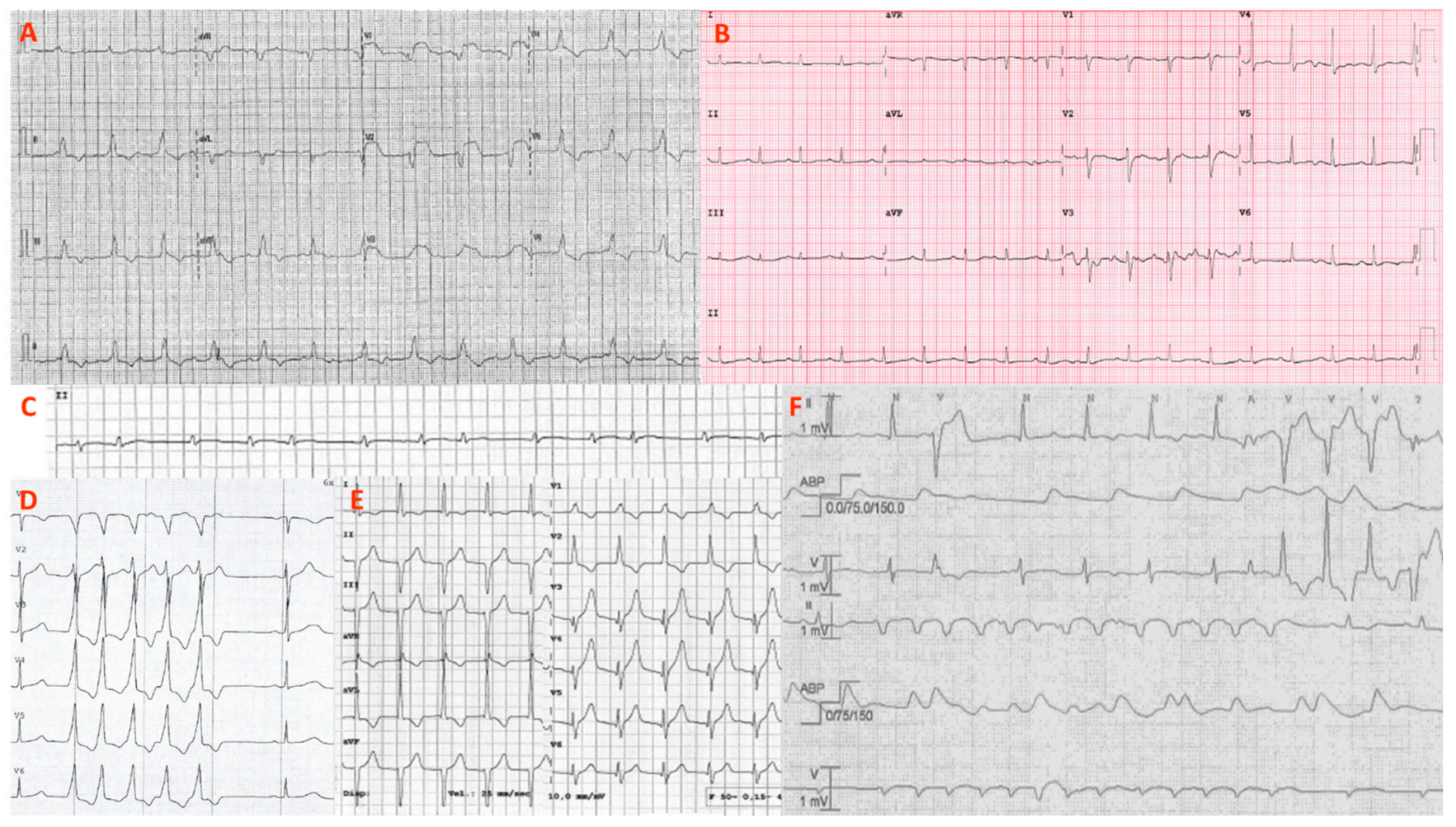

Figure 1. ECG and arrhythmias. Representative ECGs and arrhythmias from the patient series (P1-P7) are shown. (A) Atrioventricular dissociation with huge anterior ST elevation (P7); (B) sinus tachycardia with low QRS voltages and diffuse repolarization abnormalities (P2); (C) paroxysmal atrial fibrillation detected by ICU telemonitoring (P7); (D) nonsustained ventricular tachycardia (P4); (E) transitory, self-limited, accelerated junctional rhythm (P1); (F) polymorphic and irregular nonsustained ventricular tachycardias during ICU stay (P5). ICU = intensive care unit.

\subsection{Patient 2 (P2)}

A 38-year-old man was symptomatic for cough and dyspnea, but afebrile. NPS and CT were consistent with COVID-19 interstitial pneumonia. The ECG was notable for diffuse T-wave abnormalities, and the echocardiogram revealed an LVEF of $41 \%$ with septal hypokinesia and no chamber dilation. Since the T-Tn curve was unremarkable, coronary arteries were not evaluated. CMR was negative for updated Lake Louise criteria (LLC) [5] but showed septal edema. EMB documented remarkable CD68+ macrophage infiltrates, interstitial edema and spotty fibrosis. The N gene of SARS-CoV-2 was amplified from myocardial tissue, as well as low-load ( $<500$ copies of DNA/ $\mu$ g) parvovirus B19 (PV-B19) in the absence of viremia. The patient was discharged with no etiology-specific treatment. Telemedicine reassessment revealed no symptoms and an LVEF of $63 \%$ by the 6-month follow-up.

\subsection{Patient 3 (P3)}

A 58-year-old woman presented with acute chest pain after 1 week of fever up to $38.5^{\circ} \mathrm{C}$, vomit and diarrhea. She had no fever $\left(37.0^{\circ} \mathrm{C}\right)$, but the NPS tested positive for COVID-19. The ECG showed inferolateral ST-segment depression with an infarct-like T-Tn curve. The echocardiogram was unremarkable, with an LVEF of $59 \%$. The CT scan both excluded critical coronary stenosis and showed ground-glass lung consolidations, confirming COVID-19 pneumonia. CMR (Figure 2) revealed mild edema and anteroseptal late gadolinium enhancement (LGE), fulfilling criteria for acute myocarditis. On account of clinical stability and T-Tn normalization, EMB was not performed. At the 3-month follow-up, the patient had no chest pain and showed a normal echocardiogram and T-Tn. 
However, she was still symptomatic for dyspnea on effort, with desaturation up to $86 \%$ at the rapid pace walk test.

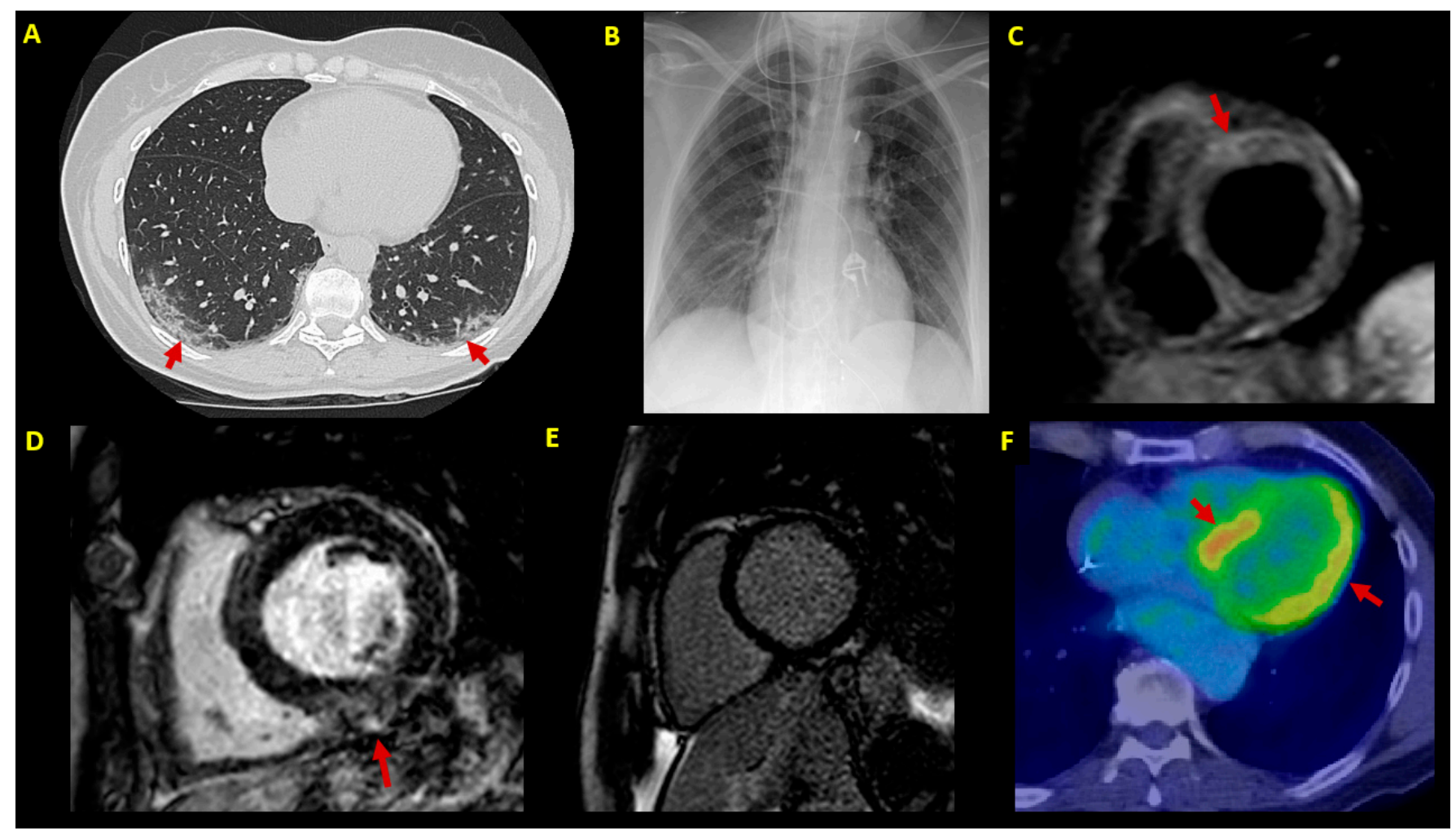

Figure 2. Imaging findings. Imaging findings at patient (P1-P7) diagnostic workup. (A) Chest CT scan showing bilateral patchy ground-glass opacities (arrows) (P3); (B) chest X-ray in a patient (P7) with cardiogenic shock supported by IABP, VA-ECMO and temporary pacemaker; (C) CMR in a patient with infarct-like acute myocarditis associated with COVID-19 (P3); T2-STIR sequence shows edema in the anterior basal segment (arrow); (D) LGE sequences in a patient (P5) showing mild inferior mid-myocardial/subepicardial LGE (arrow); (E) absence of LGE by 3-month follow-up CMR in a patient (P4) with fulminant myocarditis at presentation; (F) 3-month follow-up FDG-PET scan in an ICD carrier (P5) with virusnegative myocarditis; abnormal left ventricular FDG uptake is shown (arrows). CMR = cardiac magnetic resonance; $\mathrm{CT}$ = computed tomography; $\mathrm{EMB}=$ endomyocardial biopsy; FDG-PET=18F-fluorodeoxyglucose positron emission tomography; IABP = intra-aortic balloon pump; ICD = implantable cardioverter defibrillator; LGE = late gadolinium enhancement; LVEF = left ventricular ejection fraction; STIR = short-tau inversion recovery; VA-ECMO = venoarterial extracorporeal membrane oxygenator.

\subsection{Patient 4 (P4)}

A 45-year-old man was admitted at another institution for epigastric pain. CT showed pneumonia, and the NPS was positive for SARS-CoV-2. The echocardiogram showed an LVEF of $34 \%$ with severe biventricular dilation. The coronary CT scan was normal. Due to subsequent hemodynamic instability, the patient was transferred to our intensive care unit (ICU). HD inotropes and intra-aortic balloon pump (IABP) were necessary for 6 days. The NT-proBNP peak was 24,252 pg/mL. As the first-choice exam, EMB showed edema and myocardial inflammation. PCR analysis was negative for SARS-CoV-2, but positive for high-load PV-B19 DNA (>500 copies of DNA/ $\mu$ ). An off-label trial of daily anakinra was started. Due to premature ventricular complexes (PVCs) and nonsustained ventricular tachycardia (NSVT), the patient was discharged on metoprolol. By the 3-month follow-up, T-Tn, NTproBNP and Holter ECG were all normal. CMR showed incomplete systolic function recovery (LVEF 47\%) and mildly increased T2 mapping, with no LGE. 


\subsection{Patient 5 (P5)}

A 64-year-old male patient was admitted to our ICU following out-of-hospital cardiac arrest secondary to ventricular fibrillation. The ECG showed anterior ST elevation and the LVEF was $15 \%$. After immediate support by invasive mechanical ventilation and HD inotropes, urgent coronary angiography showed absence of culprit lesions. The lung CT scan was unremarkable, and COVID-19 was diagnosed only by repeated NPS. EMB showed virus-negative inflammatory cardiomyopathy. A few days later, CMR showed LLCproven myocarditis. Telemetry showed multiple episodes of irregular NSVT. Due to family history of sudden cardiac death, the patient underwent genotyping and dual-chamber implantable cardioverter defibrillator (ICD) placement. Furthermore, since abnormal myocardial glucose uptake was described at 3-month 18F-fluorodeoxyglucose positron emission tomography (FDG-PET), oral immunosuppressive therapy was planned but delayed because of evidence of latent tuberculosis at eligibility screening. The indication was finally withheld, since the LVEF improved up to $60 \%$ by month 6 on ACE inhibitors. ICD telemetry was uneventful.

\subsection{Patient 6 (P6)}

A 50-year-old man was admitted to an external hospital for acute dyspnea and diagnosed with COVID-19 interstitial pneumonia. The ECG showed sinus tachycardia, first-degree atrioventricular block (AVB) and low QRS voltages. At the echocardiogram, the patient had an LVEF of $20 \%$ in the absence of hemodynamic instability. T-Tn was mildly abnormal and NT-proBNP was $1122 \mathrm{pg} / \mathrm{mL}$. Coronary arteries were angiographically normal. Following multiple NSVT episodes, the patient underwent subcutaneous ICD (S-ICD) implant. Since symptomatic LV systolic dysfunction persisted at the 1-month follow-up (LVEF 19\%), elective admission on COVID-free status was planned at our institution. EMB documented virus-negative chronically active inflammatory cardiomyopathy. An immunosuppressive therapy (IST) regimen was started. However, the 6-month LVEF was $24 \%$ and the patient had persistent dyspnea. Since a preexisting dilated cardiomyopathy was suspected, the patient was both proposed for genetic testing and screened for heart transplantation.

\subsection{Patient 7 (P7)}

A 56-year-old woman with recent history of COVID-19 influential syndrome presented at another institution with oppressive chest pain and anterior ST elevation. Angiography showed normal coronaries, whereas angio-CT ruled out pulmonary embolism and aortic dissection. The echocardiogram showed severe biventricular dysfunction and right ventricular (RV) dilation. The patient was transferred to our ICU on HD inotropes, IABP and empirical iv steroids. However, cardiogenic shock was complicated by multiorgan failure, paroxysmal complete AVB and tachyarrhythmias. The patient was upgraded to venoarterial extracorporeal membrane oxygenator (VA-ECMO) support, and steroid treatment was withdrawn. EMB revealed diffuse acute lymphocytic myocarditis with massive necrosis (Figure 3). Unexpectedly, PCR analysis documented high-load Epstein-Barr virus (2500 copies of DNA/ $\mu \mathrm{g}$ ). After iv immunoglobulins and progressive weaning from circulatory support, CMR showed LVEF normalization, but persistent RV dilation $\left(97 \mathrm{~mL} / \mathrm{m}^{2}\right)$ and systolic dysfunction (RVEF $40 \%$ ) with septal edema. The patient was scheduled for heart transplantation. 


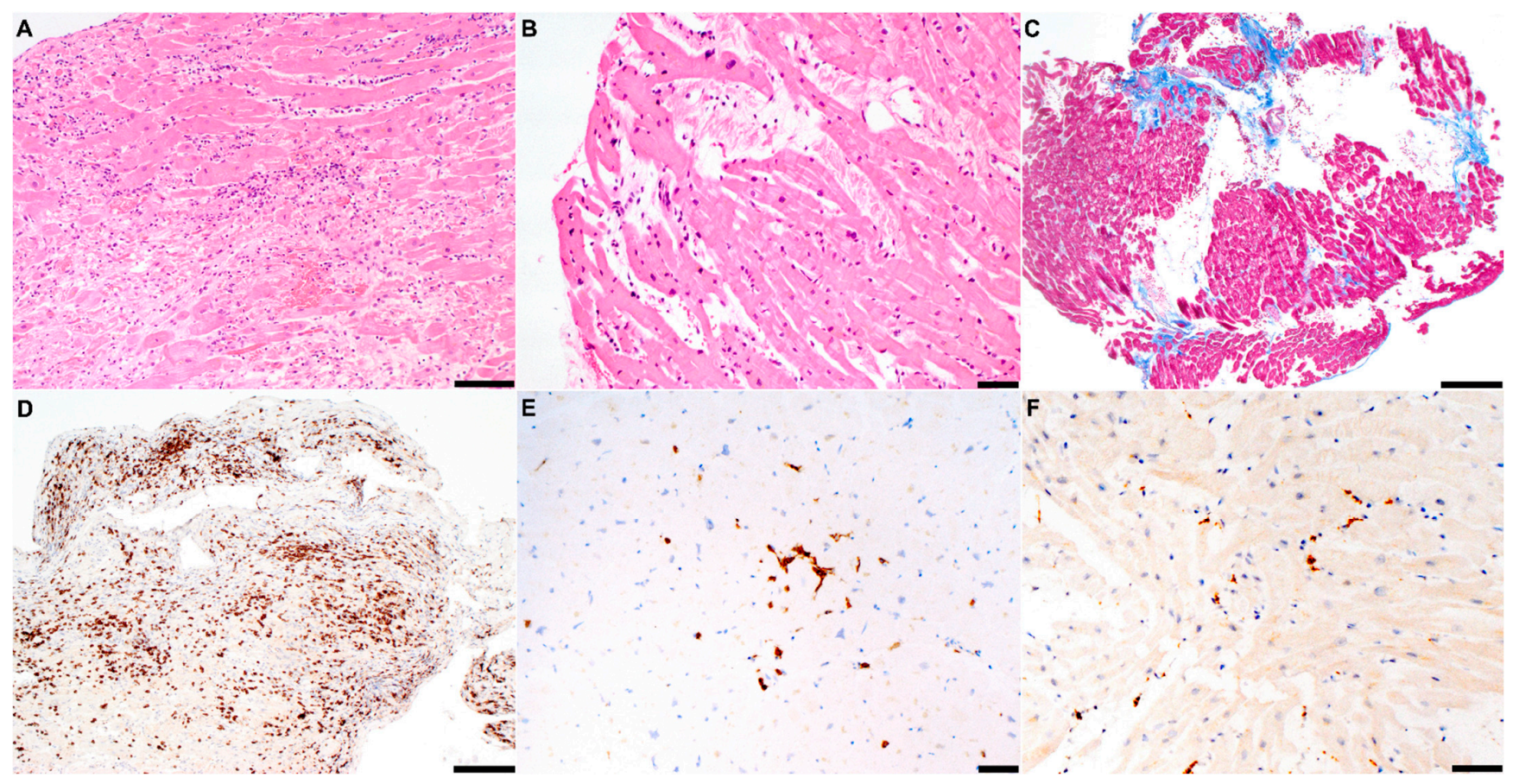

Figure 3. Histology findings. Representative findings at histologic analysis of patients (P1-P7) are shown. (A) Hematoxylineosin assay in a patient (P7) with fulminant EBV myocarditis complicating COVID-19 infection; diffuse areas of necrosis and massive lymphocytic inflammatory infiltrates are shown; (B) mild lymphocytic infiltration without significant necrosis in a patient (P1) with borderline myocarditis; (C) trichrome assay in a patient (P6) with myocarditis (not shown) and likely preexisting dilated cardiomyopathy; multiple areas of replacement fibrosis (blue) are shown; (D) immunohistochemistry for CD3+ T-lymphocytes $\left(>7 / \mathrm{mm}^{2}\right)$ in fulminant myocarditis (P7); (E) in contrast, milder CD3+ infiltrated in a patient with mild inflammatory cardiomyopathy (P5); (F) CD68+ macrophage infiltrates in a patient with SARS-CoV-2 borderline myocarditis (P2). Scale bars: (A): $100 \mu \mathrm{m}$; (B,E,F): $50 \mu \mathrm{m}$; (C,D): $200 \mu \mathrm{m}$. CD = cluster of differentiation; EBV = Epstein-Barr virus; $\mathrm{SARS}-\mathrm{CoV} 2$ = severe acute respiratory syndrome coronavirus- 2 .

\section{Discussion}

\subsection{Main Findings}

We presented a wide spectrum of clinical manifestations of COVID-19-associated myocarditis, in a series of consecutive patients undergoing diagnostic and therapeutic management worked out by a dedicated myocarditis disease unit for myocarditis [4].

\subsection{Diagnostic Workup}

Since myocarditis is often a rule-out diagnosis [6], coronary artery disease was primarily investigated in the majority of our patients. In particular, coronary angiography was performed in cases with cardiogenic shock or clinically suspected myocardial infarction (P5-7), whereas CT scan was mainly used to rule out coronary artery disease in patients with a low pre-test probability $(\mathrm{P} 1,3,4)$. This is also consistent with the algorithm proposed to investigate myocardial injury in COVID-19 patients [1,3]. In the absence of remarkable $\mathrm{T}$-Tn abnormalities, coronary imaging was spared in a young and low-risk subject (P2).

Among second-level techniques, our strategy aimed at performing both EMB and CMR in the majority of patients $(6 / 8=75 \%)$. In particular, EMB was needed to define histotype and etiology to allow disease-specific treatment [6]. Of course, EMB was a forced choice in patients with a complicated onset (P4-7, mean LVEF $25 \pm 9 \%$ ), including fulminant and arrhythmic myocarditis [6,7]. However, except for a single case with a normal LVEF and T-Tn normalization (P3), EMB was performed in all patients as the gold standard diagnostic technique $[2,6]$. Whenever possible, EMB was performed directly at bedside (P1), in compliance with the COVID-19 pandemic logistic needs [8]. As a complementary tool, CMR was chosen both to obtain panoramic heart assessment [5,9], 
and to allow follow-up disease monitoring (P4,7) [2]. Differently from EMB, CMR was the first exam in clinically stable patients with a normal or mildly reduced LVEF (P1-3, mean LVEF $48 \pm 10 \%$ ) $[9,10]$. Nonetheless, CMR was performed even in cases with fulminant myocarditis following clinical stabilization $(\mathrm{P} 4,5,7)$. As a possible alternative to CMR, to avoid ICD-related susceptibility artifacts [11], our findings support the feasibility of FDG-PET scan in patients with arrhythmic myocarditis (P5).

The overall diagnostic algorithm for COVID-19-associated myocarditis, as suggested by our experience, is summarized in Figure 4.

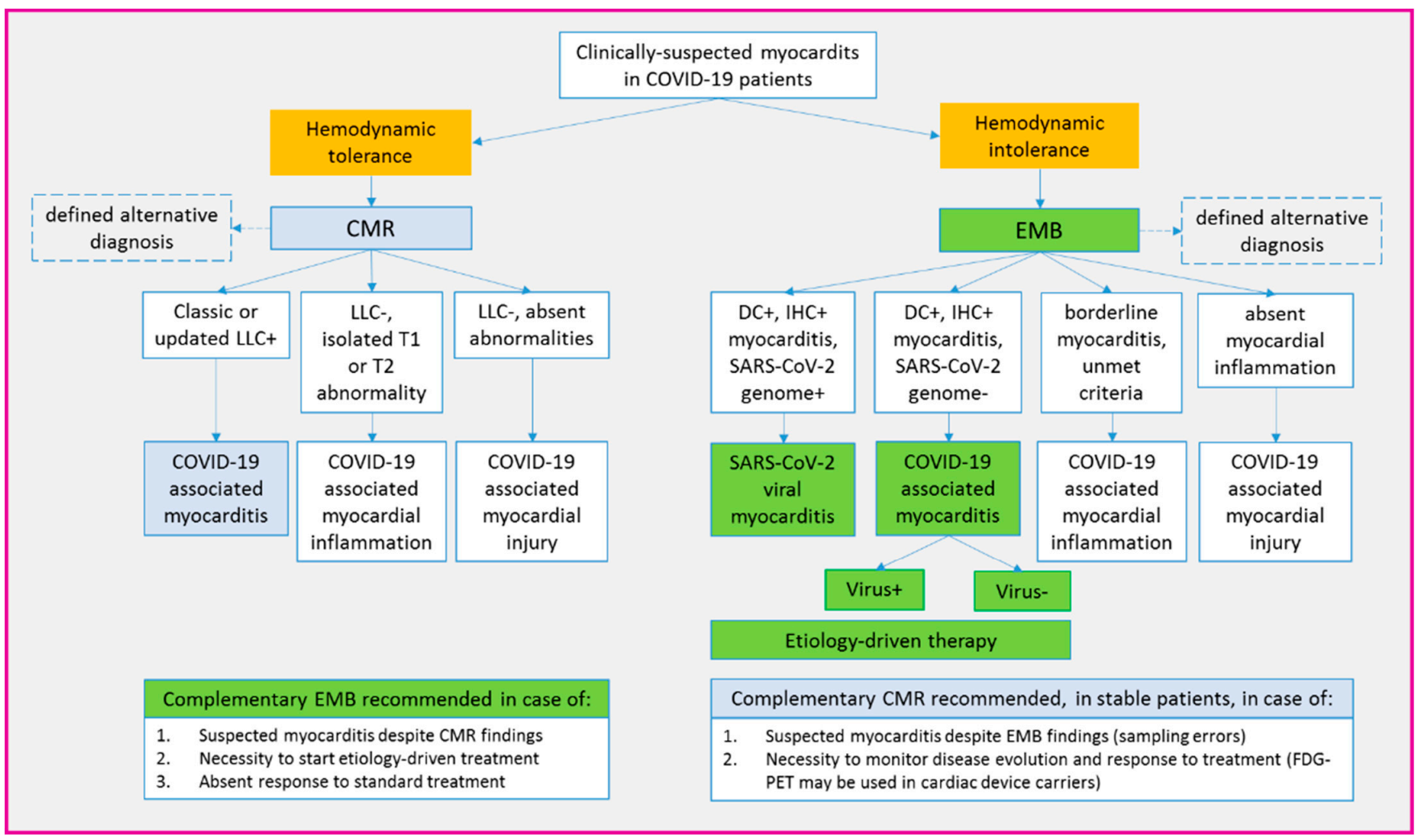

Figure 4. Proposed algorithm for diagnosis and classification of myocarditis in COVID-19 patients. Proposed diagnostic workup for patients with clinically suspected myocarditis associated with COVID-19 infection. The key criterion to guide the initial choice between CMR and EMB in hemodynamic tolerance. However, when feasible, we suggest an extensive use of both techniques, since they provide complementary information. Remarkably, based on the current definition of viral myocarditis $(2,6)$, only EMB allows for SARS-CoV-2 to be identified as responsible for myocardial inflammation. In general, etiology-driven therapy is safe only when myocarditis diagnosis is EMB-proven. CMR = cardiac magnetic resonance; $\mathrm{DC}=$ Dallas criteria $; \mathrm{EMB}=$ endomyocardial biopsy; FDG-PET=18F-fluorodeoxyglucose positron emission tomography; IHC = immunohistochemistry; LLC = Lake Louise criteria; SARS-CoV-2 = severe acute respiratory syndrome coronavirus-2.

\subsection{Disease Features and Etiology}

Diagnosis of viral myocarditis was challenging for us in COVID-19 patients, given that, by definition, myocarditis is inflammation and associated myocyte damage in a non-ischemic pattern [3]. Remarkably, the severity of myocardial involvement was often unrelated to the entity of respiratory disease. For instance, P1-3 had pneumonia and non-fulminant myocarditis, whereas P5,7 had fulminant myocarditis in the absence of pneumonia. Furthermore, the timespan between myocarditis and lung disease, as well as between prodromes and cardiac manifestations, ranged from 0 to 12 days (Table 1).

By applying the current definition of EMB-proven myocarditis [6,10], two patients (P1,7) fully met the Dallas criteria, whereas four cases (P2,4-6) had "borderline" inflammation in the absence of necrosis $[6,10]$. Consistently, although edema was uniformly 
found (Table 1), the classic LLC were inconstantly met (P3,5,7). Of course, the heterogeneous timespan between EMB and CMR (Table 1) should be acknowledged as a limiting factor. At histology, the most common inflammatory infiltrate was lymphocytic, with a relevant macrophage component in two cases $(\mathrm{P} 2,4)$. In contrast, interstitial macrophages constituted the dominant infiltration reported in a series of COVID-19 autoptic cases with myocardial inflammation in the absence of clinically suspected myocarditis [12].

Identifying myocarditis etiology was also a major issue in our series, since it provided no direct visualization of SARS-CoV-2 particles within cardiomyocytes. However, in keeping with the definition of viral myocarditis [6], SARS-CoV-2 RNA was isolated from myocardial tissue in one patient (P2): consistently with an N+/ORF1- expression pattern, which has been associated with late-phase or healing infection [13], this patient experienced a favorable clinical course. The importance of PCR analysis is also remarked by the identification of other viral genomes within myocardial samples of three patients. In particular, although a co-pathogenic role cannot be excluded for SARS-CoV-2, we reported unexpected fulminant myocarditis either by Epstein-Barr virus (P7) or PV-B19 (P4). In addition, in keeping with its doubtful pathogenic role [2,14], a low-load and likely bystander PVB-19 genome was documented in a patient (P2) coinfected with SARS-CoV-2. As for virus-negative myocarditis [6], also described in a recent series of COVID-19 autoptic cases [15], the pathophysiology is largely unexplained and deserves further elucidation. A transitory inflammatory stunning following early clearance of the SARS-CoV-2 genome can be hypothesized, given the rapid LVEF recovery documented in many patients despite the absence of IST. However, a role for autoimmunity is suggested in 4/7 AHA-positive cases [16]. The underlying pathophysiology is still to be elucidated. Differential diagnoses may also include takotsubo syndrome as in P1 [17], or preexisting underlying dilated cardiomyopathy as in P4-6, where COVID-19 infection may have just unmasked a preexisting underlying disease.

\subsection{Arrhythmias and Devices}

Although resuscitated sudden cardiac death was observed only in one case (P5), arrhythmias were documented during in-hospital monitoring in a relevant proportion of our cohort $(6 / 8=75 \%)$. In particular, ventricular arrhythmias were common among ICU patients with fulminant myocarditis (P4-7). As exemplified in Figure 1, it should be noted that ventricular arrhythmias were commonly irregular and polymorphic, hinting at an active inflammatory phase [18]. Among supraventricular arrhythmias, previously reported as the dominant manifestation of clinically stable COVID-19 patients [19], atrial fibrillation was documented only in one patient of our series (P7), in the context of cardiogenic shock. Consistently with the close spatial relationship with the conduction system [20], advanced AVB was documented only in a patient with anteroseptal disease likely unrelated to COVID-19 (P7). As a possible stress-related manifestation [17], a self-limiting episode of an accelerated junctional rhythm was also reported in a case of myocarditis overlapping with a reverse takotsubo pattern (P1).

Concerning the primary prevention of sudden death, our experience supports an ICD-sparing strategy: in fact, most of the patients in our cohort experienced a significant LVEF improvement by the time of hospital discharge (preserved LVEF in $4 / 7$ cases). Dual-chamber ICD was offered to a single case (P5) meeting both secondary and primary prevention criteria for implant: given systolic function normalization and an uneventful 6-month follow-up, however, we acknowledge that a life vest might have been applied instead, as a bridge to decision. In line with the current indications [7], S-ICD was proposed to a patient with a likely preexisting dilated cardiomyopathy and no need for pacing (P6).

\subsection{Follow-Up and Treatment}

In order to reduce the hospital inflow, a multidisciplinary telemedicine platform [4] was applied for follow-up reassessment of clinically stable patients such as P2, as well as home monitoring for all cardiac device carriers [8]. Thus far, no etiology-specific treat- 
ment has been identified for COVID-19 patients, either with or without cardiac involvement $[2,21]$. Nonetheless, a favorable clinical course was observed in the majority of cases, either spontaneously or in response to multimodal treatment including empirical drug regimens to target COVID-19 (Table 2). Remarkably, one patient with viral myocarditis (P4) underwent LVEF improvement after a 3-month treatment course by anakinra: this finding confirms the beneficial effects reported in virus-negative inflammatory cardiomyopathy [22]. In contrast, classic immunosuppression $[23,24]$ is currently contraindicated in patients with viral myocarditis [6]. Accordingly, empirical treatment by iv steroids may have accelerated the evolution towards refractory cardiogenic shock in a patient with a subsequent, unexpected diagnosis of Epstein-Barr myocarditis (P7). Our data support the importance of viral genome screening as a part of the standard assessment of any myocarditis. Additionally, absent mortality in our series suggests a relevant role for circulatory support in patients with cardiogenic shock (P4-7). Regarding EMB-proven virus-negative myocarditis, the importance of specialized immunologic evaluation and ad hoc baseline screening [25] was confirmed by the identification of latent tuberculosis as a contraindication for a safe immunosuppression in a patient (P5).

\subsection{Study Limitations}

Our data reflect the experience of a single center on a relatively small population. As a result of a patient-tailored strategy, the interval between symptom onset to diagnosis, as well as the timespan between CMR and EMB, was considerably variable; however, this reflects real-world clinical practice. Since in situ hybridization was not performed, the detection of intra-myocardial viruses was hereby limited by PCR sensitivity. Advanced diagnostic and therapeutic tools, as well as the availability of a specialized disease unit for myocarditis, make our experience difficult to be reproduced outside third-level centers. The short follow-up duration is an intrinsic study limitation.

\section{Conclusions}

We presented a patient-tailored strategy for myocarditis associated with COVID-19 infection, as assessed by a dedicated multidisciplinary disease unit at a referral center. Myocarditis diagnosis was achieved following extensive use of both multimodal imaging and $\mathrm{EMB}$, where molecular testing for viral genomes had a key role in the subsequent therapeutic choices. Although myocarditis was associated with recent-onset COVID-19 infection, alternative etiologies beyond SARS-CoV-2 were frequently documented. Our findings suggest that a hub-and-spoke model can be extensively applied during the current COVID-19 pandemic, in order to offer advanced and personalized workup, especially for patients with complicated myocarditis. Meanwhile, multicenter prospective registries are called to fill the knowledge gaps concerning characterization and outcomes of COVID-19associated myocardial inflammatory syndromes.

Supplementary Materials: The following are available online at https:/ /www.mdpi.com/article/10 $.3390 / \mathrm{jcm} 10091974 / \mathrm{s} 1$, Online Supplements.

Author Contributions: Conceptualization, G.P. and S.S. (Simone Sala); methodology, S.R., A.P., D.V., M.D.G., P.F., M.G.C. and E.F.; validation, A.M.C., M.T., F.M., L.G:, F.D.C., A.M., P.G.C. and P.M.; formal analysis, A.V. and G.P.; investigation, G.P., A.V., S.R., A.E., G.D.L., C.C., S.S. (Silvia Sartorelli), M.R., E.B. and G.C.; resources, M.T., A.M.C., F.D.C., L.G., M.S., A.M., L.D., C.B. and P.D.B.; data curation, A.V. and G.P.; writing — original draft preparation, A.V. and G.P.; writing—review and editing, S.S. (Simone Sala); visualization, S.R., A.E., G.D.L., A.P., D.V., A.M.C., M.T., C.C., S.S. (Silvia Sartorelli), M.R., M.D.G., E.B., P.F., M.G.C., E.F., F.M., G.C., L.G., F.D.C., A.M., L.D., M.S., P.G.C., P.M., P.D.B. and C.B.; supervision, P.G.C., P.D.B., P.M. and C.B. All authors have read and agreed to the published version of the manuscript.

Funding: This research received no external funding. 
Institutional Review Board Statement: The study was conducted according to the guidelines of the Declaration of Helsinki, and approved by the Institutional Review Board of San Raffaele Scientific Institute (MYOCAR, 24/01/2018).

Informed Consent Statement: Informed consent was obtained from all subjects involved in the study.

Data Availability Statement: Data will be made available, upon reasonable request, by emailing the correspondent author.

Acknowledgments: We would like to acknowledge all people that worked and keep working in our country during this pandemic. In particular, we would like to thank all nurses, anesthesiologists, infectious diseases specialists and the whole emergency personnel working at our institution.

Conflicts of Interest: The authors declare no conflict of interest.

\begin{abstract}
Abbreviations
$\mathrm{AHA}=$ anti-heart autoantibodies; $\mathrm{AVB}=$ atrioventricular block; $\mathrm{CMR}=$ cardiac magnetic resonance; $\mathrm{CT}=$ computed tomography EMB = endomyocardial biopsy; FDG-PET = 18F-fluorodeoxyglucose positron emission tomography; IABP = intra-aortic balloon pump; ICD = implantable cardioverter defibrillator; ICU = intensive care unit; LLC = Lake Louise criteria; LV = left ventricular; LVEF = left ventricular ejection fraction; NPS = nasopharyngeal swab; NSVT = nonsustained ventricular tachycardia; PV-B19 = parvovirus B19; PVCs = premature ventricular complexes; RAAS = reninangiotensin-aldosterone system; RV = right ventricular; SARS-CoV2 = severe acute respiratory syndrome coronavirus-2; T-Tn= T-troponin; $\mathrm{VA}-\mathrm{ECMO}=$ venoarterial extracorporeal membrane oxygenator; VT = ventricular tachycardia.
\end{abstract}

\title{
References
}

1. Hendren, N.S.; Drazner, M.H.; Bozkurt, B.; Cooper, L.T., Jr. Description and Proposed Management of the Acute COVID-19 Cardiovascular Syndrome. Circulation 2020, 141, 1903-1914. [CrossRef] [PubMed]

2. Tschöpe, C.; Ammirati, E.; Bozkurt, B.; Caforio, A.L.P.; Cooper, L.T.; Felix, S.B.; Hare, J.M.; Heidecker, B.; Heymans, S.; Hübner, N.; et al. Myocarditis and inflammatory cardiomyopathy: Current evidence and future directions. Nat. Rev. Cardiol. 2020, 1-25. [CrossRef]

3. Peretto, G.; Sala, S.; Caforio, A.L.P. Acute myocardial injury, MINOCA, or myocarditis? Improving characterization of coronavirusassociated myocardial involvement. Eur. Heart J. 2020, 41, 2124-2125. [CrossRef]

4. Peretto, G.; De Luca, G.; Campochiaro, C.; Palmisano, A.; Busnardo, E.; Sartorelli, S.; Barzaghi, F.; Cicalese, M.P.; Esposito, A.; Sala, S. Telemedicine in myocarditis: Evolution of a mutidisciplinary "disease unit" at the time of COVID-19 pandemic. Am. Heart J. 2020, 229, 121-126. [CrossRef] [PubMed]

5. Ferreira, V.M.; Schulz-Menger, J.; Holmvang, G.; Kramer, C.M.; Carbone, I.; Sechtem, U.; Kindermann, I.; Gutberlet, M.; Cooper, L.T.; Liu, P.; et al. Cardiovascular Magnetic Resonance in Nonischemic Myocardial Inflammation: Expert Recommendations. J. Am. Coll. Cardiol. 2018, 72, 3158-3176. [CrossRef] [PubMed]

6. Caforio, A.L.; Pankuweit, S.; Arbustini, E.; Basso, C.; Gimeno-Blanes, J.; Felix, S.B.; Fu, M.; Heliö, T.; Heymans, S.; Jahns, R. Current state of knowledge on aetiology, diagnosis, management, and therapy of myocarditis. Eur. Heart J. 2013, 34, $2636-2648$. [CrossRef]

7. Peretto, G.; Sala, S.; Rizzo, S.; De Luca, G.; Campochiaro, C.; Sartorelli, S.; Benedetti, G.; Palmisano, A.; Esposito, A.; Tresoldi, M.; et al. Arrhythmias in myocarditis: State of the art. Heart Rhythm 2019, 16, 793-801. [CrossRef] [PubMed]

8. Mazzone, P.; Peretto, G.; Radinovic, A.; Limite, L.R.; Marzi, A.; Sala, S.; Cireddu, M.; Vegara, P.; Baratto, F.; Paglino, G.; et al. The COVID-19 challenge to cardiac electrophysiologists: Optimizing resources at a referral center. J. Interv. Card Electrophysiol. 2020, 18, 1-7. [CrossRef]

9. Esposito, A.; Palmisano, A.; Natale, L.; Ligabue, G.; Peretto, G.; Lovato, L.; Vignale, D.; Fiocchi, F.; Marano, R.; Russo, V. Cardiac Magnetic Resonance Characterization of Myocarditis-Like Acute Cardiac Syndrome in COVID-19. JACC Cardiovasc. Imaging 2020. [CrossRef]

10. Ammirati, E.; Frigerio, M.; Adler, E.D.; Basso, C.; Birnie, D.H.; Brambatti, M.; Friedrich, M.G.; Klingel, K.; Lehtonen, J.; Moslehi, J.J.; et al. Management of Acute Myocarditis and Chronic Inflammatory Cardiomyopathy: An Expert Consensus Document. Circ. Heart Fail. 2020, 13, e007405. [CrossRef]

11. Palmisano, A.; Vignale, D.; Peretto, G.; Busnardo, E.; Calcagno, C.; Campochiaro, C.; De Luca, G.; Sala, S.; Ferro, P.; Basso, C.; et al. Hybrid FDG-PET/MR or FDG-PET/CT to Detect Disease Activity in Patients With Persisting Arrhythmias After Myocarditis. JACC Cardiovasc. Imaging 2020, 10. [CrossRef] [PubMed] 
12. Basso, C.; Leone, O.; Rizzo, S.; De Gaspari, M.; van der Wal, A.C.; Aubry, M.C.; Bois, M.C.; Lin, P.T.; Maleszewski, J.J.; Stone, J.R. Pathological features of COVID-19-associated myocardial injury: A multicentre cardiovascular pathology study. Eur. Heart J. 2020, 41, 3827-3835. [CrossRef] [PubMed]

13. Chu, D.K.W.; Pan, Y.; Cheng, S.M.S.; Hui, K.P.Y.; Krishnan, P.; Liu, Y.; Ng, D.Y.M.; Wan, C.K.C.; Yang, P.; Wang, Q.; et al. Molecular Diagnosis of a Novel Coronavirus (2019-nCoV) Causing an Outbreak of Pneumonia. Clin. Chem. 2020, 66, 549-555. [CrossRef]

14. Koepsell, S.A.; Anderson, D.R.; Radio, S.J. Parvovirus B19 is a bystander in adult myocarditis. Cardiovasc. Pathol. 2012, 21, 476-481. [CrossRef] [PubMed]

15. Del Nonno, F.; Frustaci, A.; Verardo, R.; Chimenti, C.; Nicastri, E.; Antinori, A.; Petrosillo, N.; Lalle, E.; Agrati, C.; Ippolito, G. Virus-Negative Myopericarditis in Human Coronavirus Infection: Report from an Autopsy Series. Circ. Heart Fail. 2020, 13. [CrossRef]

16. Caforio, A.; Mahon, N.G.; Baig, M.K.; Tona, F.; Murphy, R.T.; Elliott, P.M.; McKenna, W.J. Prospective familial assessment in dilated cardiomyopathy-Cardiac autoantibodies predict disease development in asymptomatic relatives. Circulation 2007, 115, 76-83. [CrossRef] [PubMed]

17. Sala, S.; Peretto, G.; Gramegna, M.; Palmisano, A.; Villatore, A.; Vignale, D.; De Cobelli, F.; Tresoldi, M.; Cappelletti, A.M.; Basso, C.; et al. Acute myocarditis presenting as a reverse Tako-Tsubo syndrome in a patient with SARS-CoV-2 respiratory infection. Eur. Heart J. 2020, 41, 1861-1862. [CrossRef]

18. Peretto, G.; Sala, S.; Rizzo, S.; Palmisano, A.; Esposito, A.; De Cobelli, F.; Campochiaro, C.; De Luca, G.; Foppoli, L.; Dagna, L.; et al. Ventricular Arrhythmias in Myocarditis: Characterization and Relationships with Myocardial Inflammation. J. Am. Coll. Cardiol. 2020, 75, 1046-1057. [CrossRef]

19. Sala, S.; Peretto, G.; De Luca, G.; Farina, N.; Campochiaro, C.; Tresoldi, M.; Dagna, L.; Zangrillo, A.; Gulletta, S.; Della Bella, P. Low prevalence of arrhythmias in clinically stable COVID-19 patients. Pacing Clin. Electrophysiol. 2020, 16. [CrossRef]

20. Oloriz, T.; Wellens, H.J.; Santagostino, G.; Trevisi, N.; Silberbauer, J.; Peretto, G.; Maccabelli, G.; Della Bella, P. The value of the 12-lead electrocardiogram in localizing the scar in non-ischaemic cardiomyopathy. Europace 2016, 18, 1850-1859. [CrossRef]

21. Sanders, J.M.; Monogue, M.L.; Jodlowski, T.Z.; Cutrell, J.B. Pharmacologic treatments for coronavirus disease 2019 (COVID-19): A review. JAMA 2020, 323, 1824-1836. [CrossRef]

22. De Luca, G.; Campochiaro, C.; Dinarello, C.A.; Dagna, L.; Cavalli, G. Treatment of Dilated Cardiomyopathy with Interleukin-1 Inhibition. Ann. Intern Med. 2018, 169, 819-820. [CrossRef]

23. Frustaci, A.; Russo, M.A.; Chimenti, C. Randomized study on the efficacy of immunosuppressive therapy in patients with virus-negative inflammatory cardiomyopathy: The TIMIC study. Eur. Heart J 2009, 30, 1995-2002. [CrossRef] [PubMed]

24. De Luca, G.; Campochiaro, C.; Sartorelli, S.; Peretto, G.; Dagna, L. Therapeutic strategies for virus-negative myocarditis: A comprehensive review. Eur. J. Int. Med. 2020, 77, 9-17. [CrossRef] [PubMed]

25. Peretto, G.; Sala, S.; De Luca, G.; Marcolongo, R.; Campochiaro, C.; Sartorelli, S.; Tresoldi, M.; Foppoli, L.; Palmisano, A.; Esposito, A.; et al. Immunosuppressive Therapy and Risk Stratification of Patients with Myocarditis Presenting With Ventricular Arrhythmias. JACC Clin. Electrophysiol. 2020, 6, 1221-1234. [CrossRef] [PubMed] 